\title{
APLICACIÓN DE DIFERENTES SOLUCIONES NUTRITIVAS Y DOSIS DE RIEGO EN CULTIVO DE Physalis peruviana L.
}

\author{
Gromaz, A. ${ }^{1}(\mathrm{P})$, Torres, J.F. ${ }^{2}$, Pascual Seva, $N^{3}$., San Bautista, $A .{ }^{3}$, López Galarza ${ }^{3}, \mathrm{~S}$. , \\ Pascual, $B^{3}$., Alagarda, $\mathcal{J}^{3}$., Maroto, J.V $V^{3}$. \\ ${ }^{1}$ Becario de Especialización. CVER. Universitat Politècnica de València. Camino de Vera \\ s/n, 46022 Valencia angroroi@etsia.upv.es \\ ${ }^{2}$ Investigador, CVER. Universitat Politècnica de València. jotorru@upvnet.upv.es \\ 3 Profesor, Departamento de Producción Vegetal, Universitat Politècnica de València. \\ Camino de Vera s/n, 46022 Valencia asanbau@prv.upv.es
}

\section{Resumen}

El objetivo del presente estudio fue evaluar la respuesta de las plantas de Physalis a la aplicación de diferentes dosis de riego y soluciones nutritivas, en términos productivos y de desarrollo vegetativo. Para ello, se emplearon dos dosis de riego D1 (dosis baja: $50 \% \mathrm{ET}_{\mathrm{c}}$ ) y $\mathrm{D} 2$ (dosis media: $100 \% \mathrm{ET}_{\mathrm{c}}$ ) y tres soluciones nutritivas con diferentes concentraciones de nitratos $\mathrm{S} 1\left(\left[\mathrm{NO}_{3}{ }^{-}\right]=7.91 \mathrm{mmol} \cdot \mathrm{L}^{-1}\right) ; \mathrm{S} 2\left(\left[\mathrm{NO}_{3}\right]=12.41 \mathrm{mmol} \cdot \mathrm{L}^{-1}\right)$; S3 $\left(\left[\mathrm{NO}_{3}{ }^{-}\right]=16.91 \mathrm{mmol} \cdot \mathrm{L}^{1}\right)$. Las mejores respuestas productivas se dieron con la solución $\mathrm{S} 2$ y la dosis D2 debido al mayor número de frutos formados. El aumento de la concentración de nitratos aumentó la cosecha con la dosis D1 mientras que la redujo con la dosis D2. Las plantas con un mayor peso seco de la parte aérea y mayor altura se obtuvieron con las soluciones S2 y S3 y la dosis D2. Las soluciones nutritivas empleada y la dosis de riego no ejercieron influencia en las características cualitativas de los frutos. Las mayores eficiencias de riego se consiguieron con las soluciones S2 y S3 con la dosis de riego más baja (D1).

\section{Introducción}

El alquequenje o Physalis (Physalis peruviana L.) es una especie herbácea, que se aprovecha por sus frutos comestibles, de forma globosa y de color amarillo o anaranjado, que se encuentran recubiertos por el cáliz formando una especie de farolito (Maroto,2002).

El "cracking" o rajado de los frutos es uno de los principales problemas fisiológicos que afectan la calidad y la rentabilidad en el cultivo de esta especie. Debido a su incidencia en cosecha y en poscosecha, provoca la pérdida de más del $30 \%$ de la producción (Gordillo et al., 2004) llegando a alcanzar el rechazo en la exportación, en periodos de lluvia, un valor de hasta el 50\% (Fischer, 2005). Su aparición, se ha atribuido principalmente al balance hídrico de las plantas, que afecta a la velocidad de crecimiento y la producción de frutos, siendo la variación en el contenido de humedad del suelo el principal desencadenante (Kamimura et al., 1972).

La aplicación excesiva de fertilizantes, superior a los requerimientos del cultivo, especialmente el caso del nitrógeno, puede provocar un crecimiento exuberante de las plantas, que actuaría en detrimento de la producción. En otras ocasiones, estas aportaciones no resultan perjudiciales para el rendimiento y la calidad de los frutos, pero incrementan los costos de producción y son una fuente de contaminación del suelo y acuíferos subterráneos. Por ello resulta de gran interés conocer las necesidades de la planta y establecer un protocolo de fertilización que se ajuste a las mismas (Maroto, 2009).

Uno de los factores más restrictivos en la producción hortícola es la disponibilidad de agua de riego, por ese motivo, resulta de gran importancia conocer cuáles son las 
necesidades del cultivo, y cómo afectan a la producción y a los parámetros de calidad de los frutos, la utilización de diferentes volúmenes y sistemas de riego. En cultivo sin suelo el aporte del riego debe hacerse de manera que se compensen las necesidades de la planta, evitar la acumulación de sales en el sustrato y mantener una adecuada oxigenación. Para conseguir estos objetivos, el volumen de riego aplicado debe situarse por encima de las necesidades de la planta, introduciendo un porcentaje de drenaje, situado entre el 20 o $25 \%$ del total de volumen aplicado, que servirá para llevar a cabo el lavado del exceso de sales (Baixauli y Aguilar, 2002).

En relación con esta necesidad, se han realizado distintos experimentos para determinar cuál es la respuesta productiva de las plantas al riego. Así, en experiencias llevadas a cabo con tomate (Pascual et al., 2000; Ortega-Farias et al., 2001; Ortega-Farias et al., 2003) un incremento del volumen de riego llevó asociado un aumento de la cosecha. Sin embargo, en otros estudios con tomate (Malorgio et al., 1991; San Bautista et al., 2004 a) y melón (Ribas et al., 2001; Cabello et al., 2009) se constató que, a partir de una determinada cantidad de agua aportada, no se obtenía un incremento en la producción, debido a la disminución en la eficiencia del riego.

Otro factor importante a tener en cuenta cuando se estudia la aplicación de distintas dosis de riego es establecer su influencia sobre el desarrollo vegetativo de las plantas, el cual, al ser excesivo, puede actuar en detrimento de la producción final. En ensayos con tomate (San Bautista et al., 2004 a) y melón (San Bautista et al., 2004 b) se ha obtenido que el incremento de las dosis de riego se tradujo en un mayor desarrollo vegetativo de las plantas, estimado éste en términos de altura de la planta y grosor de los tallos.

Finalmente, el riego puede afectar a la calidad final de los frutos. Así, en cultivo de tomate, el aumento del volumen de riego aplicado supuso una reducción de la firmeza de los frutos (San Bautista et al., 2004 a), del contenido en sólidos solubles (Malorgio et al., 1991; Mitchell et al., 1991; Ortega-Farias et al., 2001; 2003; San Bautista et al., 2004 a) y de la acidez de los frutos (Malorgio et al., 1991; Mitchell et al., 1991; San Bautista et al., 2004 a).

El objetivo del presente estudio fue evaluar la respuesta de las plantas de Physalis a la aplicación de diferentes dosis de riego y soluciones nutritivas, tanto en términos productivos (cantidad producida y calidad de la cosecha) como de desarrollo vegetativo.

\section{Materiales y métodos.}

Las plantas utilizadas en la experiencia, procedían de un semillero realizado el 4 de febrero de 2013, en bandejas de poliestireno de 54 alvéolos rellenas de vermiculita. La plantación definitiva en el invernadero se produjo el 11 de abril de 2013, en macetas de 35 $\mathrm{cm}$ de diámetro (25 L), empleando perlita como sustrato de cultivo, y disponiéndose una planta por contenedor.

La aportación de nutrientes se hizo a través de fertirrigación, con un sistema de riego localizado de alta frecuencia, mediante el que se aplicaban las soluciones nutritivas utilizadas. Las dosis de riego empleadas fueron:

- D1 (dosis baja): $50 \% \mathrm{ET}_{\mathrm{c}}$

- D2 (dosis media): $100 \% \mathrm{ET}_{\mathrm{c}}$

Tabla 1. Composición de las soluciones nutritivas $\left(\mathrm{mmol} \cdot \mathrm{L}^{-1}\right)$

\begin{tabular}{lllllllll}
\hline Solución nutritiva & NO3- & $\mathrm{H} 2 \mathrm{PO} 4-$ & $\mathrm{SO} 42-$ & $\mathrm{NH} 4+$ & $\mathrm{K}+$ & $\mathrm{Ca} 2+$ & $\mathrm{Mg} 2+$ & $\begin{array}{l}\mathrm{CE} \\
(\mathrm{dS} / \mathrm{m})\end{array}$ \\
\hline $\mathrm{S} 1$ & 7.91 & 1.20 & 2.45 & 0.50 & 4.00 & 3.70 & 1.46 & 1.56 \\
$\mathrm{~S} 2$ & 12.41 & 1.20 & 2.45 & 0.50 & 5.00 & 3.95 & 2.96 & 2.01 \\
$\mathrm{~S} 3$ & 16.91 & 1.20 & 2.45 & 0.50 & 6.00 & 4.20 & 4.46 & 2.46 \\
\hline
\end{tabular}


Se eligió un diseño de bloques al azar con 3 soluciones nutritivas (S1, S2 y S3), 2 dosis de riego (D1 y D2) con 3 U.R. (unidades de repoición) de 5 plantas cada una. Para cada combinación de soluciones y dosis, con un total de 18 U.R. y 90 plantas. Para aportar las distintas dosis, el sistema de riego estaba constituido por 1 gotero de $2 \mathrm{~L} \cdot \mathrm{h}-1$ por planta para la D1, y el doble para la D2.

El manejo del riego se hizo intentando mantener el porcentaje de drenaje de la dosis D1 cerca entre el $10-15 \%$ y el de la dosis D2 en valores situados entre el 30 y el $35 \%$. Se utilizó para llevar a cabo la experiencia un sistema automatizado en el que el número de riegos necesario para alcanzar el volumen de drenaje establecido se controló a través de cambios en los valores del paquete radiativo necesario para efectuar 1 riego (a igualdad de radiación, al aumentar el valor del paquete radiativo la frecuencia de riegos era menor y viceversa). Se estableció en 2 minutos la duración de cada riego. En las Tablas 2.1., 2.2. se recogen los valores (por planta) del volumen de solución aportado y porcentaje de drenaje $(\%)$.

Tabla 2.1. Volumen de solución aportado por planta $(\mathrm{mL})$

\begin{tabular}{lllllllllll}
\hline & Mayo & Junio & Julio & Agosto & Sept. & Oct. & Nov. & Dic. & Enero & Febr. \\
\hline D1 & 13400 & 41900 & 37320 & 24440 & 22550 & 14700 & 13800 & 13700 & 12600 & 10400 \\
D2 & 26800 & 83800 & 74640 & 48880 & 45100 & 29400 & 27600 & 27400 & 25200 & 20800 \\
\hline
\end{tabular}

Tabla 2.2. \% Drenaje

\begin{tabular}{lllllllllll}
\hline & Mayo & Junio & Julio & Agosto & Sept. & Oct. & Nov. & Dic. & Enero & Febr. \\
\hline S1 D1 & 13.1 & 9.3 & 19.0 & 11.5 & 14.6 & 12.1 & 15.1 & 9.5 & 7.1 & 8.7 \\
S1 D2 & 37.1 & 26.5 & 38.6 & 30.8 & 30.7 & 32.8 & 35.7 & 29.0 & 24.6 & 28.6 \\
S2 D1 & 12.9 & 9.9 & 20.0 & 10.8 & 14.9 & 11.1 & 14.1 & 12.2 & 14.6 & 11.3 \\
S2 D2 & 36.6 & 29.0 & 40.6 & 30.8 & 31.4 & 30.7 & 37.0 & 33.3 & 35.7 & 32.1 \\
S3 D1 & 12.9 & 4.8 & 19.0 & 9.5 & 14.7 & 9.4 & 15.5 & 15.8 & 20.7 & 14.8 \\
S3 D2 & 40.4 & 24.9 & 39.9 & 28.5 & 31.0 & 28.0 & 38.3 & 38.4 & 44.6 & 35.4 \\
\hline
\end{tabular}

Al finalizar el cultivo se calculó la eficiencia del riego mediante la siguiente fórmula:

Eficiencia de riego $=\frac{\text { Producción total } \mathrm{Kg}}{\mathrm{m}^{3} \text { solución nutritiva aportada }}$

Con objeto de llevar a cabo un control de la influencia de las dosis de riego sobre el desarrollo vegetativo, una vez al mes, se tomaron medidas de la altura de la planta (con una cinta métrica) y del diámetro del tallo (con un pie de rey modelo Hommel Hercules). Estas mediciones se efectuaron sobre las 5 plantas de cada repetición. Para que las medidas del grosor del tallo fueran fiables, esta medida se realizó siempre en el mismo lugar situado por encima del primer nudo, unos $2 \mathrm{~cm}$ sobre la superficie del sustrato. Además, al final del cultivo se tomó el peso fresco y seco de las hojas y de los tallos. Para realizar las medidas del peso seco de hojas y tallos se introdujeron en una estufa a $65^{\circ}$ hasta que alcanzaron un peso constante. 
Posteriormente, se tomaba de cada repetición la cantidad necesaria de frutos, para que pudieran ser triturados en un vaso de plástico con una batidora. El triturado obtenido se filtraba con un colador, realizándose una medida de ${ }^{\circ}$ Brix con un refractómetro digital ATAGO Palette PR-32. Del filtrado se tomaron $6 \mathrm{~g}$ que se introducían en un vaso de precipitado, añadiéndose agua destilada hasta alcanzar un volumen de $150 \mathrm{cc}$. El resultado fue una suspensión, que se valoró con $\mathrm{NaOH} 0.1 \mathrm{~N}$ (el punto final se alcanzaba cuando el pHmetro registraba un valor de 8.1), proceso durante el que la suspensión se mantenía en movimiento con un agitador magnético. Para el cálculo de la acidez titulable se empleó la siguiente fórmula:

$$
\mathrm{AT}(\%)=\frac{[\mathrm{mL}(\mathrm{NaOH}) \times \mathrm{N} \times 0.064] \times 100}{\text { Peso Physalis triturado }(\mathrm{g})}
$$

Donde $\mathrm{N}$ es la normalidad del $\mathrm{NaOH}$. Los resultados de acidez titulable (AT) se presentan como $\mathrm{mL}$ de ácido cítrico/100 $\mathrm{g}$ de fruto de Physalis, por ser el ácido cítrico el mayoritario de este fruto. El índice de madurez se obtuvo mediante la siguiente fórmula:

$$
\text { Índice de madurez }={ }^{\circ} \mathrm{Brix} / \mathrm{TA}(\%)
$$

El inicio de la recolección se produjo el día 11 de julio de 2013 extendiéndose hasta el 10 de febrero de 2014, con pases semanales.

\section{Resultados y discusión}

Las soluciones nutritivas con un contenido más elevado en nitratos (S2 y S3) produjeron un mayor número de frutos que la solución $S 1$ ( $p \leq 0.05)$ (Tabla 3). Sin embargo, la producción más elevada se alcanzó con la S2 ( $p \leq 0.05)$; con la solución S3 se produjo un descenso de la cosecha que aún fue más acusado con la S1 (Tabla 3). El incremento de la dosis de riego llevó a aparejado un aumento tanto del número de frutos, como de la producción final ( $p \leq 0.05 ; p \leq 0.05$ ) (Tabla 3 ).

El efecto de las soluciones nutritivas y las dosis de riego en la incidencia los distintos tipos de "cracking", no fue e.s. (Tabla 3).

\begin{tabular}{|c|c|c|c|c|c|c|}
\hline & \multirow{2}{*}{$\begin{array}{l}\text { Número } \\
\text { total de } \\
\text { frutos }\end{array}$} & \multirow{2}{*}{$\begin{array}{l}\text { Producción } \\
\text { total }(\mathrm{g})\end{array}$} & \multirow{2}{*}{$\begin{array}{c}\text { Frutos } \\
\text { comerciales } \\
(\%)\end{array}$} & \multicolumn{3}{|c|}{ Frutos afectados por fisiopatías (\%) } \\
\hline & & & & CC & $\mathrm{cl}$ & $\mathrm{cc}+\mathrm{cl}$ \\
\hline \multicolumn{7}{|l|}{ Solución } \\
\hline S1 & $148.9 \mathrm{~b}$ & $263.5 \mathrm{c}$ & $59.67-$ & $4.43-$ & $12.83-$ & $22.40-$ \\
\hline S2 & $344.9 \mathrm{a}$ & $626.5 \mathrm{a}$ & $59.83-$ & $5.25-$ & $11.17-$ & $23.48-$ \\
\hline S3 & $273.7 \mathrm{a}$ & $461.6 \mathrm{~b}$ & $60.45-$ & $2.85-$ & $16.42-$ & $20.05-$ \\
\hline \multicolumn{7}{|l|}{ Dosis de riego } \\
\hline D1 & $215.3 \mathrm{~b}$ & $374.7 \mathrm{~b}$ & $58.63-$ & $4.34-$ & $13.91-$ & $22.70-$ \\
\hline D2 & $296.4 \mathrm{a}$ & $526.4 \mathrm{a}$ & $61.33-$ & $4.01-$ & $13.03-$ & $21.26-$ \\
\hline \multirow{2}{*}{\multicolumn{7}{|c|}{\begin{tabular}{|l|l|}
\multicolumn{2}{|c|}{ Resumen ANOVA (g.I.) } \\
Factor
\end{tabular}}} \\
\hline \multicolumn{5}{|c|}{\begin{tabular}{|l|l|l} 
Factor & & \\
\end{tabular}} & & \\
\hline Solución (2) & $34.5^{\star *}$ & $36.6^{\star *}$ & $0.2 \mathrm{~ns}$ & $29.7 \mathrm{~ns}$ & $20.3 \mathrm{~ns}$ & $7.2 \mathrm{~ns}$ \\
\hline Dosis (1) & $8.6^{*}$ & $9.6^{*}$ & $3.8 \mathrm{~ns}$ & $0.8 \mathrm{~ns}$ & $0.8 \mathrm{~ns}$ & $1.8 \mathrm{~ns}$ \\
\hline \multicolumn{7}{|l|}{ Interacción } \\
\hline Sol x Dosis (2) & $38.5^{\star *}$ & $37.1^{* *}$ & $5.1 \mathrm{~ns}$ & $7.0 \mathrm{~ns}$ & $20.0 \mathrm{~ns}$ & $0.2 \mathrm{~ns}$ \\
\hline Residual (12) & 18.4 & 16.8 & 90.9 & 62.5 & 58.9 & 90.8 \\
\hline Desv. estándar & 72.5 & 123.1 & 8.1 & 1.8 & 4.6 & 6.3 \\
\hline
\end{tabular}

Tabla 3. Parámetros productivos.

Letras diferentes indican diferencias e.s. $p \leq 0.05 ;{ }^{*} y^{* *}$ indican efectos e.s. con una $p \leq 0.01$ y $p \leq 0.05$, respectivamente; 
n.s. indica que no existen diferencias e.s. La desviación estándar está expresada como la raíz cuadrada del cuadrado medio residual.

De los resultados que se recogen en la figura 1 se observa que para la dosis de riego D1 se produjo un aumento de la producción al aumentar el contenido en nitratos, lo que indicaría que, con ese volumen de riego, una solución con una mayor concentración de nitratos que la solución S3, podría haber dado lugar a una cosecha más abundante. En el caso de la dosis más alta (D2), la solución S3 ya excedía el aporte de nitratos óptimo para obtener la máxima cosecha.

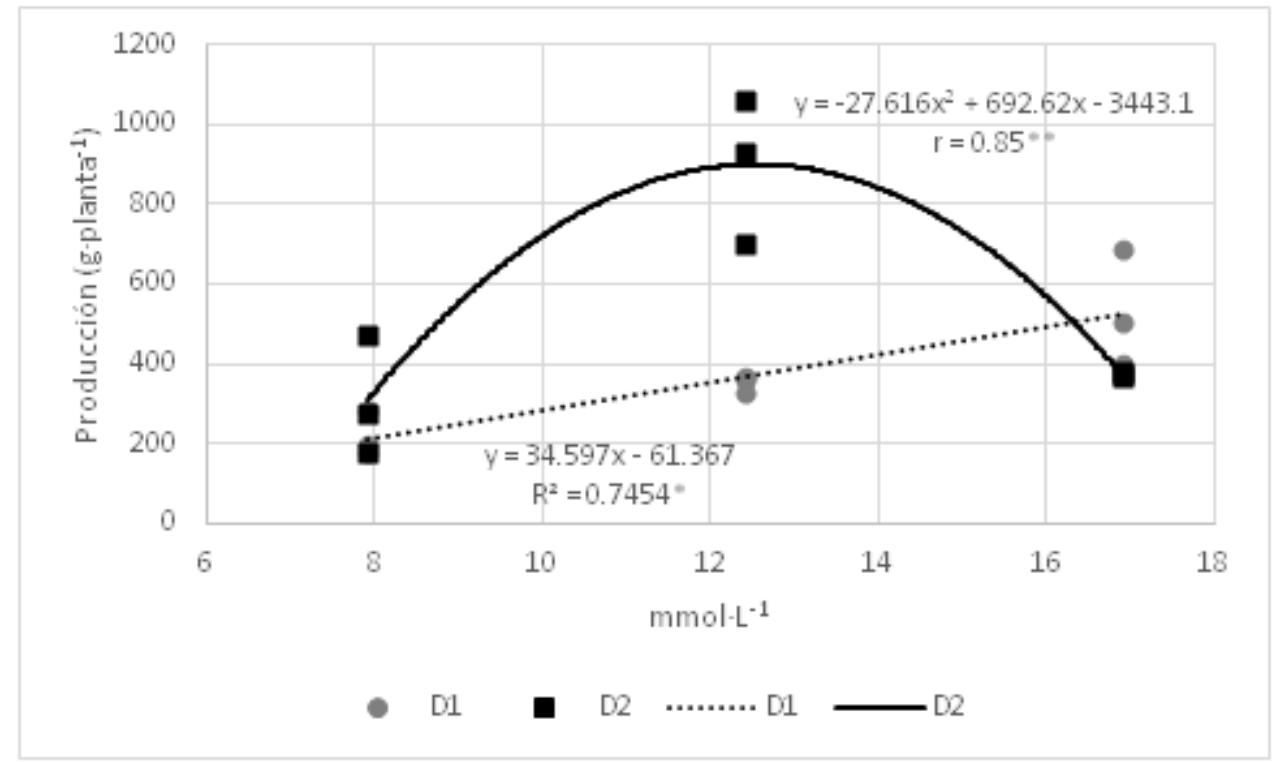

Figura 1. Producción total $\left(\mathrm{g} \cdot\right.$ planta $\left.^{-1}\right)$ para la dosis D1 y D2 frente a la concentración de $\mathrm{NO}_{3}^{-}$aportada.

Las características cualitativas de los frutos no se vieron afectadas por el efecto de las soluciones nutritivas o las dosis de riego (Tabla 4).

Tabla 4. Parámetros cualitativos de los frutos

\begin{tabular}{|c|c|c|c|c|}
\hline & Firmeza (g) & ${ }^{\circ}$ Brix & Acidez & $\begin{array}{l}\text { Índice } \\
\text { madurez }\end{array}$ \\
\hline \multicolumn{5}{|l|}{ Solución } \\
\hline $\mathrm{S} 1$ & $278.3-$ & $16.96-$ & $1.87-$ & $9.13-$ \\
\hline S2 & $280.4-$ & $16.26-$ & $1.88-$ & $8.68-$ \\
\hline S3 & $271.4-$ & $16.16-$ & $1.75-$ & $9.15-$ \\
\hline \multicolumn{5}{|l|}{ Dosis de riego } \\
\hline $\mathrm{D} 1$ & $281.4-$ & $16.64-$ & $1.87-$ & $8.91-$ \\
\hline D2 & $272.0-$ & $16.28-$ & $1.80-$ & $9.07-$ \\
\hline \multicolumn{2}{|l|}{ Resumen } & (g.I.) & \multicolumn{2}{|c|}{ \%Suma de cuadrados } \\
\hline \multicolumn{5}{|l|}{\begin{tabular}{|l|l} 
Factor \\
\end{tabular}} \\
\hline Solución (2) & $6.3 \mathrm{~ns}$ & $12.1 \mathrm{~ns}$ & $28.8 \mathrm{~ns}$ & $11.8 \mathrm{~ns}$ \\
\hline Dosis (1) & $9.4 \mathrm{~ns}$ & $3.2 \mathrm{~ns}$ & $9.1 \mathrm{~ns}$ & $1.5 \mathrm{~ns}$ \\
\hline \multicolumn{5}{|l|}{ Interacción } \\
\hline Sol $x$ Dosis (2) & $19.8 \mathrm{~ns}$ & $0.6 \mathrm{~ns}$ & $1.5 \mathrm{~ns}$ & $1.4 \mathrm{~ns}$ \\
\hline Residual (12) & 64.5 & 84.1 & 60.6 & 85.2 \\
\hline Desv. estándar & 15.1 & 1.1 & 0.1 & 0.7 \\
\hline
\end{tabular}


estándar está expresada como la raíz cuadrada del cuadrado medio residual.

Las soluciones nutritivas que aportaban más cantidad de nitratos (S2 y S3) dieron lugar a un mayor desarrollo vegetativo $(p \leq 0.05)$, que se puso de manifiesto en una mayor altura de las plantas $(p \leq 0.05)$, así como por un incremento del peso seco de la parte aérea $(p \leq 0.05)$ (Tabla 5). Entre las soluciones nutritivas, la S2 y S3 presentaron la mayor eficiencia de riego ( $p \leq 0.05)$, en tanto que el empleo de la dosis de riego más alta (D2) disminuyó la eficiencia respecto a la dosis D1 ( $\leq \leq 0.05)$ (Tabla 5).

Tabla 5. Parámetros de crecimiento vegetativo y riego

\begin{tabular}{|c|c|c|c|c|}
\hline & Altura $(\mathrm{cm})$ & $\begin{array}{l}\text { Diámetro } \\
\text { tallo }(\mathrm{mm})\end{array}$ & $\begin{array}{l}\text { PS parte } \\
\text { aérea }(\mathrm{g})\end{array}$ & $\begin{array}{l}\text { Eficiencia de } \\
\text { riego }\left(\mathrm{Kg} / \mathrm{m}^{3}\right)\end{array}$ \\
\hline \multicolumn{5}{|l|}{ Solución } \\
\hline S1 & $167.3 \mathrm{~b}$ & $10.97-$ & $512.4 \mathrm{~b}$ & $0.911 \mathrm{~b}$ \\
\hline S2 & $202.9 a$ & $11.94-$ & $798.6 \mathrm{ab}$ & $1.961 \mathrm{a}$ \\
\hline S3 & $192.9 \mathrm{a}$ & $12.17-$ & $1063.9 \mathrm{a}$ & $1.799 a$ \\
\hline \multicolumn{5}{|l|}{ Dosis de riego } \\
\hline $\mathrm{D} 1$ & $169.0 \mathrm{~b}$ & $11.59-$ & $612.7 \mathrm{~b}$ & $1.829 \mathrm{a}$ \\
\hline D2 & $206.5 \mathrm{a}$ & $11.80-$ & $970.6 \mathrm{a}$ & $1.285 \mathrm{~b}$ \\
\hline Resumen & ANOVA & (g.I.) & \%Suma de & cuadrados \\
\hline \multicolumn{5}{|l|}{ Factor } \\
\hline Solución (2) & $28.0^{*}$ & $9.0 \mathrm{~ns}$ & $39.5^{\star *}$ & $33.6^{\star \star}$ \\
\hline Dosis (1) & $40.9^{\star *}$ & $0.4 \mathrm{~ns}$ & $27.3^{\star \star}$ & $11.7^{*}$ \\
\hline \multicolumn{5}{|l|}{ Interacción } \\
\hline Sol $x$ Dosis (2) & $3.2 \mathrm{~ns}$ & $24.3 \mathrm{~ns}$ & $3.8 \mathrm{~ns}$ & $34.2^{\star \star}$ \\
\hline Residual (12) & 15.8 & 65.3 & 17.9 & 20.5 \\
\hline Desv. estándar & 14.7 & 1.7 & 183.5 & 0.4 \\
\hline
\end{tabular}

Los resultados obtenidos pusieron de manifiesto que la solución con un contenido intermedio de nitratos ( $\mathrm{S} 2=12.41 \mathrm{mmol} / \mathrm{L})$ mejoró la producción obtenida con la solución de menor concentración $(\mathrm{S} 1=7.91 \mathrm{mmol} / \mathrm{L})$, mientras que la solución $\mathrm{S} 3(16.91 \mathrm{mmol} / \mathrm{L})$, con la mayor concentración de este anión, no consiguió mejorar la producción de la S2, alcanzando un valor algo inferior. Estos resultados coinciden con los obtenidos en ensayos realizados con pimiento, en el que la producción más elevada se logró con concentraciones de nitratos en la solución nutritiva de $9 \mathrm{mmol} / \mathrm{L}$, ocasionándose descensos en la cosecha con concentraciones superiores (Bat-Tal et al, 2001 a). En cultivo de pepino dulce, cantidades de nitratos superiores a los $8 \mathrm{mmol} / \mathrm{L}$ tampoco llevaron asociada ninguna mejora productiva (Torres, 2001), lo que pone de manifiesto la gran complejidad que implica establecer el abonado nitrogenado óptimo para un cultivo, ya que los factores ambientales, la fase de desarrollo en la que se encuentra la planta, e incluso, el comportamiento varietal, pueden inducir respuestas no deseadas. Uno de los riegos de llevar a cabo un abonado con nitrógeno excesivo, es que se puede inducir en las plantas un crecimiento exuberante, que actuaría en detrimento de la producción. Los datos de los parámetros vegetativos obtenidos al final del cultivo indicaron que al aumentar la concentración de nitratos en la solución nutritiva se produjo un incremento del peso seco de la parte aérea, lo cual podría explicar por qué la solución con más nitratos no sólo no aumentó la producción, sino que, incluso, llegó a disminuirla. Las soluciones nutritivas influyeron sobre la cosecha a través del número de frutos obtenido, ya que el peso medio de los mismos no presentó variaciones al final del experimento (Dato no mostrado). 
Tal y como se ha constatado en otros cultivos hortícolas como el tomate (Pascual et al., 2000; Ortega-Farias et al, 2001; Ortega-Farias et al, 2003) y melón (Ribas et al., 2003; San Bautista et al., 2004 b), el aumento de la dosis de riego, indujo en las plantas de Physalis, al final del cultivo, un incremento de la producción. Es habitual que el incremento de la producción obtenido al aumentar la dosis de riego se produzca como consecuencia de la obtención de frutos con un mayor peso medio, tal y como se ha constatado en experiencias con tomate (Pascual et al., 2000; San Bautista et al., 2004 a) o melón (Ribas et al., 2003; San Bautista et al., 2004 b; Cabello et al., 2009), sin embargo, en la actual experiencia, el incremento en la producción, fue consecuencia del aumento del número de frutos obtenido, pero no del peso medio.

A priori, existen dos importantes factores, como la C.E. (Conductividad Eléctrica) de las soluciones nutritivas y la influencia de los nitratos en el desarrollo vegetativo de las plantas, a través de los cuales, las soluciones nutritivas podrían incidir en la aparición de "cracking" en los frutos. En lo que respecta al primer factor, el empleo de soluciones con una C.E. más alta puede producir la disminución de rajado en los frutos como consecuencia del descenso del potencial hídrico (baja el potencial osmótico) que restringiría la entrada de agua en el fruto, tal y como se ha recogido en experimentos llevados a cabo en tomate (Chrétien et al., 2000). Posiblemente, las diferencias de C.E. existentes entre las distintas soluciones nutritivas empleadas no permitieron ejercer una influencia clara sobre la aparición de esta fisiopatía. Por otra parte, un exceso de nitratos puede provocar la formación de una masa foliar exuberante y un bajo número de frutos, causando un desequilibrio en la planta que disminuiría la competencia por los fotoasimilados, los cuales ejercerían una mayor presión en el interior de los frutos, haciéndolos más susceptibles al rajado. En el presente experimento, el incremento en peso de la parte aérea asociado al aumento de nitratos en las soluciones no implicó una mayor incidencia de rajado, ya que no se encontraron diferencias significativas en los porcentajes de frutos afectados para las soluciones nutritivas y dosis de riego.

En cuanto al riego, la aportación de una dosis más alta, provocaría un incremento del potencial hídrico que facilitaría la entrada de agua en el fruto haciéndolo más susceptible al "cracking", tal y como se recoge en experimentos realizados con Physalis (Gordillo et al., 2004) y tomate (Peet y Willits, 1995; Ortega-Farias et al., 2003). En el actual experimento esta circunstancia no ha podido ser constatada. Por su parte, el mayor desarrollo vegetativo provocado por la dosis D2 tampoco incidió decisivamente en una disminución del rajado.

Se ha constatado un incremento de la altura de las plantas al aumentar la dosis de riego, lo cual coincide con lo obtenido por San Bautista et al. (2004 a) para tomate.

Las mayores eficiencias de riego se lograron con las soluciones S2 y S3, ya que el incremento en la concentración de nitratos hace que, en igualdad de dosis de riego, se obtenga una mayor producción. También se observa que el aumento de concentración de la solución S3 respecto a la S2, no indujo mejora en la eficiencia.

En cuanto a las dosis de riego, la más baja (D1) produjo una mayor eficiencia, posiblemente debido a que con la dosis D2 se produjo un crecimiento vegetativo excesivo que actuó en detrimento de la cosecha, lo cual coincide con lo obtenido en experimentos llevados a cabo con pimiento (Mohamed Yahadih Ahmed, 2005).

\section{Conclusiones}

La solución S2 y la dosis D2 fueron las que dieron las mejores respuestas productivas.

También se determinó que con la dosis $\mathrm{D} 1$, el incremento de nitratos de las soluciones aumentó la cosecha obtenida, mientras que para la dosis D2, la solución con mayor contenido en nitratos redujo la producción.

La mayor cosecha obtenida con las soluciones S2 y la dosis D2 se debió al mayor número de frutos formado, ya que ninguna de ellas afectó al peso medio de los frutos. 
Las soluciones nutritivas y dosis de riego empleadas no ejercieron una influencia clara en la aparición de rajado en los frutos.

Las soluciones S2 y S3 y la dosis D2 dieron las plantas con un mayor peso seco de la parte aérea y con una mayor altura.

Las soluciones nutritivas y las dosis de riego no ejercieron una influencia clara en las características cualitativas de los frutos.

Las mayores eficiencias de riego se consiguieron con las soluciones S2 y S3 y con la dosis de riego más baja (D1).

\section{Bibliografía}

Baixauli, C. y Aguilar, J.M. (2002). Cultivo sin suelo de hortalizas: aspectos prácticos y experiencias. Divulgación Técnica: 53. Generalitat Valenciana Consellería d'Agricultura, Peixca i Alimentació. España. 110 p.

Bar-Tal, A., Aloni, B., Karni, L. y Rosenberg, R. (2001). Nitrogen nutrition of greenhouse pepper. II. Effects of nitrogen concentration and NO3:NH4 ratio on yield, fruit Shape and the incidence of blossom-end rot in relation to plant mineral composition. Hortscience. 36 (7): 1252-1259

Cabello, M.J., Castellanos, M.T., Romojaro, F., Martínez-Madrid, C. y Ribas, F. (2009). Yield and quality of melon grown under different irrigation and nitrogen rates. Agricultural Water Management. 96 (5): 866-874

Chrétien, S. and Gosselin, A. (2000). High Electrical Conductivity and Radiation-based Water Management Improve Fruit Quality of Greenhouse Tomatoes Grown in Rockwool. Hortscience 35(4):627-631.

Fischer, G. 2005. El problema del rajado del fruto de uchuva y su posible contol. En: Avances en cultivo, poscosecha y exportación de la uchuva (Physalis peruviana L.) en Colombia. Eds G. Fischer, W. Piedrahita, D. Miranda. J. Romero. Universidad Nacional de Colombia. Facultad de agronomía. Bogotá. pp 55-82

Gordillo, O.P., Fischer, G y Guerrero, R. (2004). Efecto del riego y de la fertilización sobre la incidencia del rajado en frutos de uchuva (Physalis peruviana L.) en la zona de Silvana (Cundinamarca). Agronomia Colombiana 22 (1): 53-62

Kamimura, S., Yoshikawa, H.Ito and K. Ito. 1972. Studies on fruit cracking in tomatoes. Bulletin of the Horticultural research station of the Ministry of Agriculture and Forestry №. 7, Serie C. Morioka.

Malorgio F., Pardossi A., Tognoni F., Bertolacci M., Casarotti D., Martignon G y Schiavi M. (1991). Controllo del consumo idrico nella coltivazione senza suolo del pomodoro. Colture protette, 8/9: 123-127.

Maroto, J.V.(2002). Horticultura Herbácea Especial. Ediciones Mundi-Prensa. Madrid 702 pp.

Maroto, J.V. (2008). Elementos de Horticultura General. Ediciones Mundi-Prensa. Madrid. $475 \mathrm{pp}$.

Mitchell, J.P., Shennan, C., Grattan, S.R. y May, D.M. (1991). Tomato fruit yields and quality under water deficit and salinity. J. Amer. Soc. Hort. Sci. 116 (2): 215-221

Mohamed Yahadih Ahmed. (2005). Influencia de diversas técnicas hortícolas en los parámetros productivos (cuantitativos y cualitativos) y el comportamiento agronómico del cultivo forzado de pimiento (Capsicum annuum L.). Tesis doctoral E.T.S.I.A. U.P.V.

Ortega-Farías, S., Márquez, J., Valdés, H. y Paillán, J.H. (2001). Efecto de cuatro láminas de agua sobre el rendimiento y calidad de tomates (Lycopersicon esculentum Mill. cV. FA144) de invernadero producido en otoño. Agricultura Técnica 61 (4): 479-487.

Ortega-Farias, S., Leyton, B, Valdés, H y Paillán, H. (2003). Efecto de cuatro láminas de agua sobre el rendimiento y calidad de tomates de invernadero producido en primavera-verano. Agricultura Técnica (Chile) 63 (4): 394-402 
Pascual, B., Maroto, J.V., San Bautista, A., López-Galarza y Alagarda, J. (2000). Influence of watering on the yield and cracking of cherry, fresh-market and processing tomatoes. Journal of Horticultural Science \& Biotechnology 75 (2): 171-175

Peet, M.M. y Willits, D.H. (1995). Role of excess water in tomato fruit cracking. HortScience 30:65-68

Ribas, F., Cabello, M. J., Moreno, M. M., Moreno, A. y López-Bellido, L. (2001). Influencia del riego y de la aplicación de potasio en la producción del melón (Cucumis melo L.). I: Rendimiento. Invest. Ag: Prod. Prot. Veg. 16 (2): 283-297

Ribas, F., Cabello, M. J., Moreno, M. M., Moreno, A. y López-Bellido, L. (2003). Influencia del riego y de la aplicación de potasio en la producción del melón (Cucumis melo L.) II. Calidad. Spanish journal of agricultural research, 1 (1): 79-90

San Bautista, A., López-Galarza, S., Pascual, B., Alagarda, J., Bono, M., Palau, V., Laza, P. y Maroto, J.V. (2004 a). Producción e incidencia de fisiopatías como respuesta a diferentes dosis de riego y soluciones nutritivas en dos cvs de tomate bajo invernadero. Cuadernos de fitopatología $3^{\text {er }}$ trimestre 85-93

San Bautista, A., López-Galarza, S., Torres, J., Bono, M.S., Laza, P., Palau, V., Solís, G., Pascual, B., Alagarda, J. y Maroto, J.V. (2004 b). Efecto de diversas dosis de riego sobre la producción, calidad, incidencia de fisiopatías, y parámetros del desarrollo en cultivo protegido de melón. Agrícola vergel: Fruticultura, horticultura, floricultura. Año 23; $275: 562-569$

Torres, J.F. (2001). Influencia de diversas técnicas de cultivo sobre los principales parámetros productivos de varios clones de pepino dulce (Solanum muricatum Ait.). Trabajo Fin de Carrera. E.T.S.I.A. U.P.V. 